\title{
COMPLEMENTARY ANALYSIS OF THE \\ INITIAL VALUE PROBLEM \\ FOR A SYSTEM OF O.D.E. MODELLING THE \\ IMMUNE SYSTEM AFTER VACCINATIONS
}

Abstract. Complementary analysis of a model of the human immune system after a series of vaccinations, proposed in [7] and studied in [6], is presented. It is shown that all coordinates of every solution have at most two extremal values. The theoretical results are compared with experimental data.

1. Introduction. In this paper, we prove the theorem which was presented in [6] without proof. The model proposed in [7] and studied in [6], and in this paper, is a modification of Marchuk's model ([1], [11], [12]) of the immune system. High concentration of antibodies after a series of vaccinations is taken into account. The idea of modifications to Marchuk's model is based on the simple one-dimensional model proposed in [2]. Immune processes in such a physical situation were described in [8]-[10].

Let $V(t), C(t), F(t)$ denote the concentrations of antigens, plasma cells and antibodies, respectively, at time $t$. We study the model

$$
\left\{\begin{array}{l}
\dot{V}=-\gamma F V, \\
\dot{C}=\alpha V F-\mu_{c}\left(C-C^{*}\right), \\
\dot{F}=\varrho C-\mu_{f} F-\nu\left(F-F^{*}\right)^{2}-\eta \gamma V F,
\end{array}\right.
$$

with non-negative coefficients. The interpretation of the equations and coefficients is the following:

- The antigens injected in vaccination are not able to reproduce. Therefore, the number of antigens depends only on the suppression by antibodies.

1991 Mathematics Subject Classification: 34C35, 34D05, 34A50.

Key words and phrases: antigen, antibody, plasma cell, B-cell, VT-complex, lymphocyte, ordinary differential equations, phase space, stationary state, stability. 
- $\gamma$ is a coefficient expressing the probability of the antigen-antibody meeting and their interactions.

- Stimulation of B-cells (which are some kind of immune cells) by VTcomplexes (which are structures built on the basis of antigens and lymphocytes) is a trigger of the plasma cell production process (to simplify the model, it is assumed that the VT-complex rate depends on the number of antigen-antibody meetings).

- $\alpha$ is an immune process stimulation coefficient.

- The plasma cell production decreases with the increasing deviation from the physiological level, denoted by $C^{*}$.

- $\mu_{c}$ is a plasma cell coefficient, with $\mu_{c}^{-1}$ equal to the mean plasma cell lifetime.

- In normal physical situation, the number of antigens depends on their production rate and death due to immune processes and natural ageing.

- $\varrho$ is the antibody production rate per plasma cell.

- $\eta$ is the rate of antibodies necessary to suppress one antigen.

- $\mu_{f}$ is an antibody coefficient, with $\mu_{f}^{-1}$ equal to the mean antibody lifetime.

- In the situation considered in this paper, there is a very high level of antibodies in the organism. This causes additional mortality of antibodies.

- $\nu$ is a coefficient of additional mortality of antibodies.

The model defined by (1) with the initial condition $\left(V_{0}, C_{0}, F_{0}\right)$,

$$
V_{0} \geq 0, \quad C_{0} \geq C^{*}, \quad F_{0} \geq 0,
$$

will be referred to as $\mathrm{VCN}$ (see [7]). We assume that

$$
2 \nu F^{*}<\mu_{f},
$$

where $F^{*}=\varrho C^{*} / \mu_{f}$ is the physiological level of antibodies. (2) means that the density coefficient $\nu$ is small compared with the antibody coefficient $\mu_{f}$ (and it is the real situation). We also assume that

$$
\alpha \varrho>\eta \gamma \mu_{c},
$$

which means that the immune system is efficient. For example, (3) is satisfied in the case of large immune process stimulation coefficient.

Define

$$
\kappa=\alpha \varrho-\eta \gamma \mu_{c} .
$$

By $(3), \kappa>0$.

2. Qualitative analysis. Setting

$$
c(t)=C(t)-C^{*}, \quad \phi(t)=F(t) V(t)
$$


yields

$$
\left\{\begin{array}{l}
\dot{V}=-\gamma \phi \\
\dot{c}=\alpha \phi-\mu_{c} c, \\
\dot{F}=\varrho c-\mu_{f}\left(F-F^{*}\right)-\nu\left(F-F^{*}\right)^{2}-\eta \gamma \phi .
\end{array}\right.
$$

In [7], it was proved that

- there exists a unique and non-negative solution of $\mathrm{VCN}$, for every $t>0$;

- if (2) and (3) hold, then VCN has a unique stationary state.

Let $\bar{X}=\left(0, C^{*}, F^{*}\right)$ denote the unique stationary state of VCN. It was also proved that every solution $X(t)$ of $\mathrm{VCN}$ has a limit as $t \rightarrow \infty$, and

$$
\lim _{t \rightarrow \infty} X(t)=\bar{X}
$$

In [6], we presented a preliminary analysis of the phase space $(F, c)$ of (4). In the case of solutions of $\mathrm{VCN}$, the functions $F$ and $c$ are functionals of $V(t)$ (see [4], [5]). We know that (see [6], [7])

$$
V(t) \rightarrow 0 \quad \text { as } t \rightarrow \infty .
$$

Let $I_{1}$ and $I_{2}$ denote the isoclines at time $t$. Then

$$
\begin{aligned}
& I_{1}=\left\{(F, c): c=\frac{\alpha V}{\mu_{c}} F\right\}, \\
& I_{2}=\left\{(F, c): c=\frac{1}{\varrho}\left[\nu\left(F-F^{*}\right)^{2}+\mu_{f}\left(F-F^{*}\right)+\eta \gamma V F\right]\right\} .
\end{aligned}
$$

The isoclines $I_{1}$ and $I_{2}$ have one common point $(\bar{F}, \bar{c})$, for every $t>0$.

Let $R_{1}, R_{2}, R_{3}, R_{4}$ denote the regions limited by the curves $I_{1}, I_{2}$ and the lines $c=0, F=0$, as in Figure 1 .

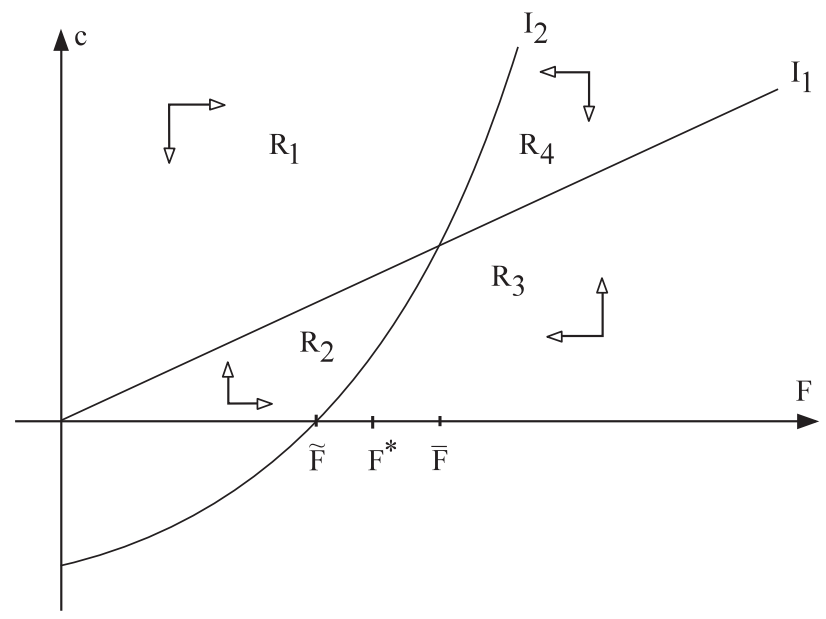

Fig. 1. VCN phase space 
Let $\Gamma(t)$ denote an arbitrary trajectory of $\mathrm{VCN}$, i.e.

$$
\Gamma(t)=\{(F(t), c(t)): t \geq 0\} .
$$

In [6], it was proved that only some kinds of behaviour of $\Gamma$ are possible:

- $\Gamma$ can pass from $R_{3}$ to $R_{4}$.

- $\Gamma$ can pass from $R_{3}$ to $R_{2}$.

- $\Gamma$ can pass from $R_{2}$ to $R_{1}$.

- $\Gamma$ can pass from $R_{1}$ to $R_{2}$.

- $\Gamma$ can pass from $R_{1}$ to $R_{4}$.

- $\Gamma$ can pass from $R_{4}$ to $R_{1}$.

- If $\Gamma$ passes from $R_{2}$ to $R_{1}$, then it cannot return to $R_{2}$.

Now, we prove the following

THEOREM 1. If the trajectory $\Gamma$ passes from the region $R_{1}$ to $R_{4}$, then it cannot return to $R_{1}$.

Proof. Assume that the trajectory passes from $R_{1}$ to $R_{4}$, and next returns to $R_{1}$. Then there exists an interval $\left(t_{0}, \bar{t}\right)$ such that $\Gamma(t) \in R_{4}$ for $t \in\left(t_{0}, \bar{t}\right), \Gamma(t) \in R_{1}$ for $t<t_{0}$, and $\Gamma\left(t_{0}\right) \in I_{2}, \Gamma(\bar{t}) \in I_{2}$. In this case,

$$
F(\bar{t}) \geq \bar{F}, \quad c(\bar{t}) \geq \bar{c} .
$$

Assume that $F(\bar{t})=\bar{F}$ and $c(\bar{t})=\bar{c}$. The functions $V(t), c(t), F(t)$ are decreasing on the interval $\left(t_{0}, \bar{t}\right)$, and

$$
\begin{aligned}
V\left(t_{0}\right) & >V(t)>V(\bar{t}), \\
c\left(t_{0}\right) & >c(t)>\bar{c}, \\
F\left(t_{0}\right) & >F(t)>\bar{F} .
\end{aligned}
$$

Therefore,

$$
\begin{aligned}
\bar{c} & =c\left(t_{0}\right) e^{-\mu_{c}\left(\bar{t}-t_{0}\right)}+\alpha e^{-\mu_{c} \bar{t}} \int_{t_{0}}^{\bar{t}} e^{\mu_{c} s} V(s) F(s) d s \\
& >c\left(t_{0}\right) e^{-\mu_{c}\left(\bar{t}-t_{0}\right)}+\frac{\alpha}{\mu_{c}} V(\bar{t}) \bar{F}\left(1-e^{-\mu_{c}\left(\bar{t}-t_{0}\right)}\right) .
\end{aligned}
$$

The point $(\bar{F}, \bar{c})$ is in $I_{1}$, hence $\bar{c}=\frac{\alpha}{\mu_{c}} V(\bar{t}) \bar{F}$. Therefore, $\bar{c}>c\left(t_{0}\right)$, which contradicts $(7)$.

Now, assume that $F(\bar{t})>\bar{F}$ and $c(\bar{t})>\bar{c}$. We show that there exists an interval $(\widehat{t}, \bar{t})$ on which

$$
\dot{F} \leq \frac{\kappa}{\alpha \mu_{c}} \dot{c} .
$$

The definitions of $t_{0}, \bar{t}$ are such that $F$ has a local maximum at $t_{0}$, and a local minimum at $\bar{t}$. Therefore, $\ddot{F}\left(t_{0}\right) \leq 0$ and $\ddot{F}(\bar{t}) \geq 0$. Hence, there exists $t_{1} \in\left(t_{0}, \bar{t}\right)$ such that 


$$
\ddot{F}\left(t_{1}\right)=0, \quad \ddot{F}(t) \begin{cases}\geq 0 & \text { for } t \in\left[t_{1}, \bar{t}\right], \\ \leq 0 & \text { for } t<t_{1} .\end{cases}
$$

This means that $\ddot{F}$ is increasing at $t_{1}$, and then

$$
\frac{d^{3} F}{d t^{3}}\left(t_{1}\right) \geq 0
$$

One has

$$
0 \leq \ddot{F}(t)=\varrho \dot{c}-\eta \gamma \dot{\phi}-\mu_{f} \dot{F}-2 \nu\left(F-F^{*}\right) \dot{F} \quad \text { for } t \in\left[t_{1}, \bar{t}\right] .
$$

Therefore,

$$
\dot{F} \leq \frac{\varrho \dot{c}-\eta \gamma \dot{\phi}}{\mu_{f}+2 \nu\left(F-F^{*}\right)} .
$$

To show (8), one needs to compare $\dot{c}$ and $\dot{\phi}$. Assume that

$$
\ddot{c}\left(t_{1}\right) \leq 0 .
$$

Then

$$
\begin{aligned}
\frac{d^{3} F}{d t^{3}}\left(t_{1}\right)= & \varrho \ddot{c}\left(t_{1}\right)-\eta \gamma \ddot{\phi}\left(t_{1}\right)-\mu_{f} \ddot{F}\left(t_{1}\right)-2 \nu\left(\dot{F}\left(t_{1}\right)\right)^{2} \\
& -2 \nu\left(F\left(t_{1}\right)-F^{*}\right) \ddot{F}\left(t_{1}\right) \\
< & -\eta \gamma \ddot{\phi}\left(t_{1}\right) \\
= & -\eta \gamma\left[\ddot{V}\left(t_{1}\right) F\left(t_{1}\right)+2 \dot{V}\left(t_{1}\right) \dot{F}\left(t_{1}\right)+V\left(t_{1}\right) \ddot{F}\left(t_{1}\right)\right] \\
= & -\eta \gamma\left[-\gamma F\left(t_{1}\right)\left(\dot{V}\left(t_{1}\right) F\left(t_{1}\right)+V\left(t_{1}\right) \dot{F}\left(t_{1}\right)\right)+2 \dot{V}\left(t_{1}\right) \dot{F}\left(t_{1}\right)\right] .
\end{aligned}
$$

The functions $V$ and $F$ are decreasing at $t_{1}$, hence (12) implies that

which contradicts $(9)$.

$$
\frac{d^{3} F}{d t^{3}}\left(t_{1}\right)<0
$$

Therefore, $\ddot{c}\left(t_{1}\right)>0$. We show that

$$
\ddot{c}(t)>0 \quad \text { for } t \in\left[t_{1}, \bar{t}\right] .
$$

If not, then there exists $t_{2} \in\left(t_{1}, \bar{t}\right)$ such that $\ddot{c}\left(t_{2}\right)=0$ and $\ddot{c}(t)>0$ for $t \in\left[t_{1}, t_{2}\right)$. Hence the function $\ddot{c}(t)$ decreases at $t_{2}$, i.e.

$$
\frac{d^{3} c}{d t^{3}}\left(t_{2}\right) \leq 0
$$

But

$$
\begin{aligned}
\frac{d^{3} c}{d t^{3}}\left(t_{2}\right)= & \alpha \ddot{\phi}\left(t_{2}\right) \\
= & \alpha\left[\ddot{V}\left(t_{2}\right) F\left(t_{2}\right)+2 \dot{V}\left(t_{2}\right) \dot{F}\left(t_{2}\right)+V\left(t_{2}\right) \ddot{F}\left(t_{2}\right)\right] \\
= & \alpha\left[-\gamma F\left(t_{2}\right)\left(\dot{V}\left(t_{2}\right) F\left(t_{2}\right)+V\left(t_{2}\right) \dot{F}\left(t_{2}\right)\right)\right. \\
& \left.+2 \dot{V}\left(t_{2}\right) \dot{F}\left(t_{2}\right)+V\left(t_{2}\right) \ddot{F}\left(t_{2}\right)\right] .
\end{aligned}
$$


Since $\dot{V}\left(t_{2}\right)<0, \dot{F}\left(t_{2}\right)<0$ and $\ddot{F}\left(t_{2}\right) \geq 0$, we have

$$
\frac{d^{3} c}{d t^{3}}\left(t_{2}\right)>0
$$

which contradicts (14). Hence such a $t_{2}$ does not exist, and $\ddot{c}(t)>0$ for $t \in\left[t_{1}, \bar{t}\right]$, which implies that

$$
\frac{\alpha}{\mu_{c}} \dot{\phi}>\dot{c} .
$$

Using (10), we obtain

$$
\dot{F} \leq \frac{\kappa}{\alpha}\left(\mu_{f}+2 \nu\left(F-F^{*}\right)\right) .
$$

Therefore, (8) is satisfied, and then

$$
\dot{F}(\bar{t})<\frac{\kappa}{\alpha} \dot{c}(\bar{t})<0,
$$

which contradicts the assumption that $F$ has a minimum at $\bar{t}$.

Hence such a $\bar{t}$ does not exist, and the trajectory $\Gamma$ stays in $R_{4}$.

3. Conclusions and applications. The propositions of [6] and Theorem 1 prove that there are only five possible types of behaviour of solutions of $\mathrm{VCN}$ :

1. $F$ is decreasing, $C$ has one maximum.

2. $F$ has one minimum and one maximum, $C$ has one maximum.

3. $F$ has one maximum, $C$ has one minimum and one maximum.

4. $F$ is increasing, $C$ has one minimum and one maximum.

5. $F$ has one minimum, $C$ has one maximum.

Only types 1 and 2 concern the case $F_{0}>F^{*}$, i.e. describe the behaviour after a series of vaccinations. There are exactly two types of behaviour which are observed after a series of vaccinations - either the concentration of antibodies decreases to $F^{*}$, or it reaches its maximal value and next decreases.

In [7], we have compared solutions of VCN with the experimental data published in [8]. Now, it occurs that one can better fit the solution to those data.

In the case of vaccinations, VCN reduces to the following model (for details see [7]):

$$
\dot{c}=-\mu_{c} c, \quad \dot{f}=\varrho c-\mu_{f} f-\nu f^{2},
$$

where $c=C-C^{*}, f=F-F^{*}$.

We compare solutions of the $\mathrm{VCN}$ with the following experimental data. Let $t_{i}$ denote the months after the last vaccination when the levels of antibodies were measured, and $F_{i}$ denotes the values of measurements (in \%). 
TABLE 1. Parameters for (16) that yield a local minimum of mean-square error between the solution of this system and experimental data

\begin{tabular}{cl}
\hline Parameter & Value \\
\hline$\varrho$ & 0.32609 \\
$\mu_{c}$ & 0.005509 \\
$\mu_{f}$ & 0.100309 \\
$\nu$ & 0.001991 \\
\hline
\end{tabular}

TABLE 2. Comparison between experimental data $F_{i}$ and the solution $f\left(t_{i}\right)$ of (16) for estimated parameters (see Table 1)

\begin{tabular}{cccl}
\hline$t_{i}$ & $F_{i}$ & \multicolumn{1}{c}{$f\left(t_{i}\right)$} & \multicolumn{1}{c}{ Error } \\
\hline 0 & 100 & 100 & 0 \\
17 & 8.91 & 8.753663 & 0.156337 \\
24 & 4.91 & 5.351945 & 0.441945 \\
36 & 3.72 & 3.304686 & 0.415314 \\
48 & 2.62 & 2.6704 & 0.0504 \\
81 & 2.04 & 2.108001 & 0.068001 \\
\hline
\end{tabular}

Then the solution of (16) with parameters shown in Table 1 leads to the result and local errors shown in Table 2 and Figures 2, 3, and to the global error

$$
\sum_{i}\left(F_{i}-f\left(t_{i}\right)\right)^{2}=0.399407
$$

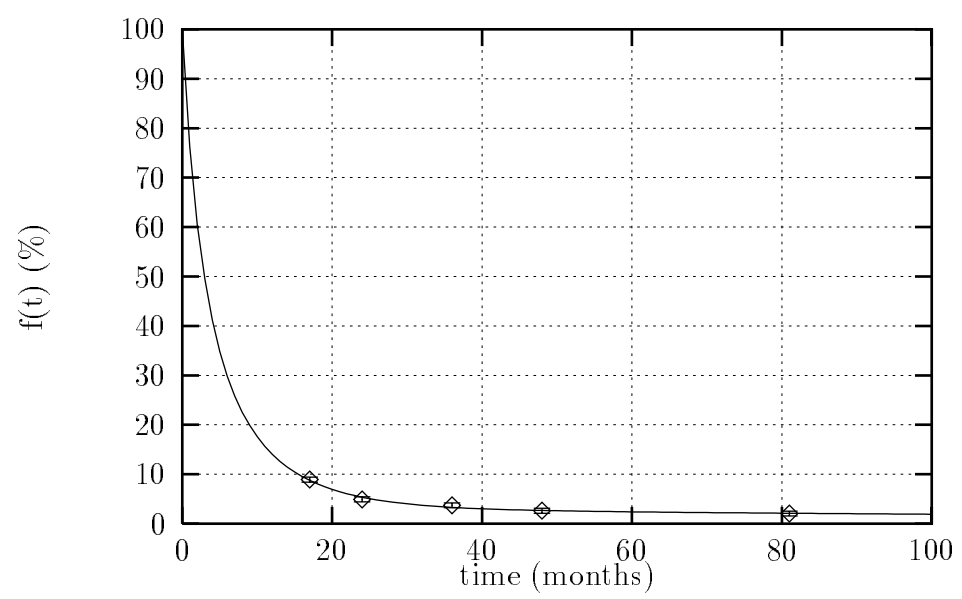

Fig 2. Comparison between experimental data and the solution of (16) on the interval $[0,100]$

This quality of approximation has been attained by first fitting the analytical solution of (16) with fixed $\varrho=0$ to experimental data. Then pa- 
rameters calculated in this way were used as a starting point to looking for better fit when $\varrho \neq 0$. To solve numerically general equations (16) we used the CVODE package [3].

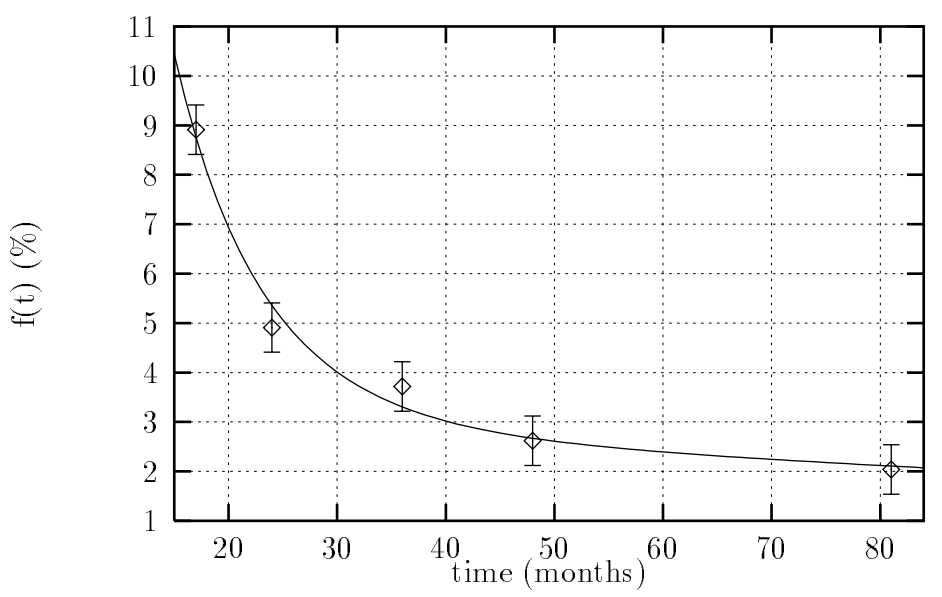

Fig. 3. Comparison between experimantal data and the solution of (16) on the interval $[10,90]$

Acknowledgements. The authors wish to express their thanks to the Interdisciplinary Centre for Mathematical and Computational Modelling (ICM UW) for access to hardware and software resources.

\section{References}

[1] L. N. Belykh, Analysis of Mathematical Models in Immunology, Nauka, Moscow, 1988 (in Russian).

[2] A. Borkowska and W. Szlenk, A mathematical model of decreasing of antibodies concentration after vaccination in the case of hepatitis B, Polish J. Immunology 20 (1995), 117-122.

[3] S. D. Cohen and A. C. Hindmarsh, CVODE, a Stiff/Nonstiff ODE Solver in C, Computers in Physics 10 (1996), no. 2.

[4] U. Foryś, Global analysis of Marchuk's model in a case of weak immune system, Math. Comput. Modelling 25 (1997), 97-106.

[5] - Global analysis of Marchuk's model in case of strong immune system, J. Math. Biol., to appear.

[6] - Global analysis of the initial value problem for a system of O.D.E. modelling the immune system after vaccinations, Math. Comput. Modelling 29 (1999), 79-85.

[7] U. Foryś and N. Żołek, A model of immune system after vaccinations, ARI 50 (1998), 180-184.

[8] M. Gesemann and N. Scheiermann, Kinetics of hepatitis B vaccine-induced anti-hbs antibodies during 82 month post-booster period, in: Proc. Internat. Sympos. Viral and Liver Disease, Tokyo, 1993, abs. 244. 
[9] A. J. Hall, Immunization against viral hepatitis type B: how long protection and against what?, Brit. Med. 1994, IV 7-8.

[10] K. Madaliński, Vaccination against hepatitis B-Current status and perspectives, Polish J. Immunology 20 (1995), 3-15.

[11] G. I. Marchuk, Mathematical Models in Immunology, Optimization Software, Publ. Division, New York, 1983.

[12] - Mathematical Modelling of Immune Response in Infectious Diseases, Kluwer Acad. Publ., Dordrecht, 1997.

Urszula Foryś

Department of Mathematics,

Informatics and Mechanics

Institute of Applied Mathematics

Warsaw University

Banacha 2, 02-097 Warszawa, Poland

E-mail: urszula@mimuw.edu.pl
Norbert S. Żołek Institute of Biocybernetics and Biomedical Engineering Trojdena 4, 02-109 Warszawa, Poland

Received on 15.1.1999;

revised version on 6.5.1999 